\title{
EFICACIA DE UN PROGRAMA DE APOYO PSICOLÓGICO A MUJERES INMIGRANTES: UN ESTUDIO DE CASOS
}

\author{
Karmele Salaberría, Analía SÁnchez y PAZ de CORRAL \\ Facultad de Psicología, Universidad del País Vasco
}

\begin{abstract}
Resumen: El objetivo de este estudio era comprobar la eficacia de un programa de apoyo psicológico en mujeres inmigrantes dirigido a paliar la sintomatología ansioso-depresiva-somática y confusional derivada del estrés migratorio. La muestra consta de 5 casos que fueron evaluados antes de recibir el tratamiento, al finalizarlo y en los seguimientos de 1 y 3 meses. El programa de apoyo psicológico cognitivo-conductual consta de 8 sesiones semanales individuales de hora y media de duración. Los resultados ponen de manifiesto la utilidad del programa, con una mejoría de los síntomas psicopatológicos y un logro de las conductas objetivo entre la evaluación pretratamiento y el postratamiento, y un mantenimiento de esta mejoría entre el postratamiento y el seguimiento de los 3 meses. Se comentan las implicaciones de este estudio para la investigación y la práctica clínica.
\end{abstract}

Palabras clave: Mujeres inmigrantes, estrés migratorio, psicopatología, apoyo psicológico.

Efficacy of a psychological support program for women immigrants: A cases study

\begin{abstract}
The goal of this study was to verify the efficacy of a psychological support program for women immigrants, directed at reducing anxious-depressive and somatic symptoms and confusion resulting from migratory stress. The sample consisted of 5 cases that were evaluated before receiving treatment, at its end and at 1 and 3 months. The cognitive-behavioral psychological support program consisted of 8 individual weekly sessions of 90 minutes duration. The results confirm the utility of the program, with an improvement of the psychopathological symptoms and a change to the behavioral objective between pre and post-treatment, maintaining the improvement between posttreatment and 3 months follow-up. Implications of this study for future research and for clinical practice are discussed.
\end{abstract}

Keywords: Women immigrants, migratory distress, psychopathology, psychological support.

\section{INTRODUCCIÓN}

Los movimientos migratorios forman parte de la historia de la humanidad, pero a comienzos del siglo XXI la sociedad se encuentra cara a cara con el fenómeno de la inmigración globalizada.

Recibido: 18-marzo-2009; aceptado: 30-junio-2009.

Correspondencia: Facultad de Psicología, Universidad del País Vasco, Avda. Tolosa, 70, 20018 San Sebastián. Correo-e: mcarmen.salaberria@ehu.es

Agradecimientos: Este trabajo se ha financiado por medio de un proyecto de investigación de la Universidad del País Vasco EHU 06/08. Asimismo, agradecemos la colaboración de instituciones de ayuda a los inmigrantes (Cruz Roja, Caritas, SOS-Racismo, Heldu y Técnico de inmigración del Ayuntamiento de San Sebastián).
Se habla de inmigrantes para referirse a todas aquellas personas extranjeras que entran en el territorio nacional con el propósito de mejorar o cambiar una situación ocasionada por causas políticas, religiosas o étnicas (refugiado o asilado) o económicas y con el objeto de escapar de una persecución o mejorar su nivel de vida, dentro de un proyecto laboral o de reagrupación familiar (Colegio Oficial de Psicólogos, 1994).

De acuerdo con el avance provisional del Padrón municipal a 1 de enero de 2008 del Instituto Nacional de Estadística (www.ine.es), del total de personas empadronadas, 40,84 millones corresponden a personas de nacionalidad española y 5,22 millones son extranjeros. La proporción de ciudadanos extranjeros es del $11,3 \%$. Entre los extranjeros el 53,2\% son hom- 
bres y el $46,8 \%$ son mujeres. La mayoría, el $63,9 \%$, tiene entre 16 y 45 años. Entre los extranjeros residentes en España, los pertenecientes a la Unión Europea-27 son 2,1 millones, de los que la mayoría son ciudadanos rumanos, británicos y alemanes. Entre los no comunitarios, destacan los marroquíes, seguidos de los ecuatorianos y los colombianos. Los ciudadanos de América del Sur suponen un 29,5\% de los extranjeros y entre ellos hay mayor proporción de mujeres que de hombres. Dentro de la Comunidad Autónoma Vasca (CAPV), según datos del año 2007 del Observatorio Vasco de inmigración (www.ikuspegi.org), los extranjeros suponen un $4,6 \%$ de la población de la comunidad y en concreto en Guipúzcoa un 4,2\%.

Desde un punto de vista psicológico, la emigración supone afrontar tres tareas principales: a) elaborar duelos y pérdidas de lo dejado en el país de origen (Salvador Sánchez, 2001); b) hacer frente a múltiples situaciones de estrés y supervivencia (Hovey, 2001); y c) adaptarse a una nueva cultura y crearse una nueva identidad (Villar, 2002). Así, el proceso migratorio se podría considerar un estrés crónico, ya que supone una sobrecarga permanente o de larga duración y que puede despertar una reacción emocional intensa y/o un esfuerzo para adaptarse de larga duración (Talarn, Navarro, Rosselle y Rigat, 2006).

Desde el punto de vista de la identidad, la inmigración pone en duda la conciencia del yo a lo largo del tiempo, la consistencia entre el yo y el mundo externo y la confirmación de la propia identidad en interacción con el ambiente, lo que supone un reto a la coherencia, la confianza y el control de uno mismo (GarzaGuerrero, 1974). La inmigración pone en duda las respuestas a la pregunta que nos hacemos todos acerca de ¿quién soy yo? (Walsh, Shulman, Feldman y Maurer, 2005), ya que los inmigrantes experimentan inestabilidad y múltiples contradicciones en sus vidas, que les llevan a reconstruir su sentido del yo y a crear nuevos significados (Mallona, 1999).

Según Achotegui (2000) y García-Campayo (2000), el proceso migratorio supone la elaboración de duelos por la pérdida de elementos importantes para la vida de las personas: la pérdida de la red social (amigos y familia), la identidad (la lengua, la cultura, la tierra, el grupo de pertenencia), el estatus social, el contacto con el grupo étnico y la integridad física (por los riesgos físicos ligados a la inmigración). Estos duelos tienen como característica que no son definitivos y que además se reavivan periódicamente.

Así, emigrar supone un proceso de aculturación que ha sido estudiado desde la psicología social y que da lugar a cuatro procesos principales (Berry, 2001), derivados, por un lado, del deseo de relacionarse con la nueva cultura y, por otro, del deseo de mantener la identidad y cultura original: a) marginación: separación de la cultura original y de la receptora; b) separación: mantenimiento de la propia cultura y evitación de la receptora; c) asimilación: abandono de la propia cultura a favor de la receptora; y por último d) integración o biculturalismo: mantenimiento de la propia identidad y relación con la cultura receptora. Es este último proceso el que correlaciona con un mejor estado psicológico, pero requiere de esfuerzos por parte del inmigrante y también por parte del país receptor, que no siempre está dispuesto a respetar y crear identidades multiétnicas y una sociedad multicultural. La aculturación es un proceso de cambio psicológico y cultural que se da a nivel grupal e individual y supone modificaciones en las estructuras sociales, en las instituciones y en las prácticas culturales; y a nivel individual, cambios en el repertorio conductual de las personas (Berry, 2005).

En un estudio reciente realizado con 182 inmigrantes residentes en España (Ramos y León, 2007), la mayoría de la muestra estudiada (un $82,2 \%$ ) se inclinaba por la integración en la sociedad receptora: esa era su aspiración. Los mismos resultados aparecen en el estudio realizado en la Comunidad Autónoma Vasca en el año 2004, donde el 74\% de los inmigrantes optaban por la integración (Basabe, Zlobina y Paéz, 2004).

Según Tizón (1994), para que el proceso migratorio se pueda elaborar de modo satisfactorio tienen que darse una serie de fases: un primer período de asentamiento, seguido de uno de adaptación, para poder llegar finalmente al de la integración. En cada una de las fases existen múltiples tareas que los inmigrantes deben realizar, a distintos niveles. En la primera fase de asentamiento deben buscar y mantener un 
trabajo, deben encontrar vivienda, deben cumplir con obligaciones económicas y legales (obtención de "papeles") aquí y con la familia dejada en el país de origen (Alma, 1986). Para encarar todas estas tareas la principal herramienta que tiene el inmigrante es su estado de salud física y mental y sus recursos psicológicos.

En diversos estudios se ha encontrado que el estrés en el emigrante aumenta entre los tres y cinco primeros años, principalmente por la escasez de apoyo social (Flaherty, Kohn, Levav y Birz, 1988), y posteriormente, años después del asentamiento, cuando emergen los problemas familiares con los hijos (por reagrupamiento familiar o por nacimiento en el país receptor), cuando hay malas condiciones de salud y cuando persisten las dificultades económicas (Lerner, Kertes y Zilber, 2005; Pernice y Brook, 1996; Ritsner y Ponizovsky, 1999; Ritsner, Ponizovsky and Ginath, 1997). En el estudio realizado por Martínez-Taboada, Arnoso y Elgorriaga (2006) se describen tres etapas en el proceso migratorio: una primera de acogida donde los inmigrantes presentan grandes necesidades de asistencia social; una segunda de adaptación sociolaboral; y una última de autonomía social. Es en la primera etapa - donde los inmigrantes llevan menos de cinco años en el país receptorcuando se presentan mayor número de síntomas ansiosos, somáticos y depresivos.

Desde el punto de vista de la salud mental, y en la primera fase de asentamiento, los procesos de aculturación y los esfuerzos para la adaptación suponen un aumento del estrés y de la sintomatología ansioso-depresiva (Hovey y Magaña, 2000). Asimismo, se sabe que el estrés y su modo de afrontamiento tienen correlaciones con la aparición de síntomas somáticos (González y Landero, 2007). En España hay algunos estudios realizados sobre la salud de los inmigrantes. Así, Jansá y García Olalla (2004) señalan en un estudio realizado en Barcelona en el año 1997 que un $48 \%$ de los hombres inmigrantes y un $65,7 \%$ de las mujeres declaraban tener un estado de salud regular, malo o muy malo. En el estudio realizado por Valiente, Sandín, Chorot, Santed y González de Rivera (1996) los inmigrantes muestran mayor nivel de psicopatología que los no inmigrantes, evaluados por medio del SCL90-R (Derogatis, 1975).
García-Campayo y Sanz (2002) señalan que los problemas más frecuentes que se presentan en la población inmigrante son el trastorno de estrés postraumático en refugiados y asilados, así como la ansiedad, la depresión, las somatizaciones y, en los casos más graves, la esquizofrenia y la paranoia. Achotegui $(2002,2003)$ ha señalado que la sintomatología del inmigrante se caracteriza por síntomas depresivos, ansiosos, confusionales y somatizaciones (dolores de cabeza, dolores abdominales, intensa fatiga y problemas de sueño). Esta sintomatología está directamente relacionada con el nivel de estrés crónico, múltiple e intenso y con el escaso apoyo social, así como con la situación legal que padecen los inmigrantes. Otros autores señalan la posibilidad de que los inmigrantes presenten más trastornos adaptativos (Matamala y Crespo, 2004) y mayores tasas de violencia doméstica y juego patológico (Petry, Armentano, Kuoch, Norinth y Smith, 2003; Steele, Lemieux-Charles, Clark y Glazier, 2002).

En el proceso de adaptación a la nueva cultura y sociedad se han detectado diversos factores de riesgo y protectores de la salud, que pueden ayudar o dificultar la adaptación. Así, la mayor distancia cultural, la situación de irregularidad, las características disfuncionales de la familia de origen, la existencia de trastornos psicológicos previos, la baja autoestima, los problemas en la salud física, una mayor edad, un menor nivel de instrucción, la baja religiosidad y la escasez de redes de apoyo social dificultan la adaptación a la nueva cultura y sociedad y facilitan la aparición de problemas y trastornos psicológicos (García-Campayo y Sanz, 2002; Hovey y Magaña, 2000; Jarvis, Kirmayer, Weinfeld y Lasry, 2005; Martínez, García y Maya, 1999; Pumariega, Rothe y Pumariega, 2005; Scott y Scott, 1985).

A pesar de los datos precedentes, apenas existen programas de apoyo psicológico estructurados para inmigrantes (Bernal, 2002) y los que existen combinan un enfoque ecléctico (dinámico-cognitivo) (Salvador Sánchez, 2001) con arteterapia (Marsen, 2004). En estos trabajos no se describen los instrumentos de evaluación, las técnicas utilizadas con detalle y no se presenta la evolución de los resultados a largo plazo. Teniendo en cuenta esta situación se 
elaboró un programa de apoyo psicológico de orientación cognitivo-conductual para que sirviera como un dispositivo de atención primaria que cubriera necesidades emocionales de apoyo, ayuda, comprensión y evitara la aparición o la cronicidad de los síntomas, ayudando al inmigrante a organizar su vida relacional y social, a adaptarse a su nueva situación (Marsella y Pedersen, 2004; Ritsner, Ponizovsky y Ginath, 1997), $y$ hacer frente a multitud de factores estresantes (Kareem y Littlewood, 2000). En este trabajo se presentan los resultados de la eficacia de este programa de apoyo psicológico en cinco mujeres inmigrantes.

\section{MÉTODO}

\section{Participantes}

La muestra de este estudio está compuesta por 5 mujeres inmigrantes latinoamericanas (2 hondureñas, 1 boliviana, 1 colombiana y 1 venezolana) que solicitaron cita en el Programa de Apoyo Psicológico a Inmigrantes de la Universidad del País Vasco. Todas ellas eran residentes en Guipúzcoa. Los criterios de admisión para este estudio han sido los siguientes: a) que el paciente sea mayor de 18 años; b) que desee participar voluntariamente en el programa después de haber sido informado; c) que sea capaz de completar los cuestionarios y tenga un mínimo de conocimiento del castellano; y d) desde el punto de vista psicopatológico que no presente un trastorno psicótico, un trastorno adictivo, un trastorno afectivo grave con ideación suicida, un trastorno de alimentación, ni una enfermedad crónica grave.

Las participantes tenían una edad media de 28,6 años (rango 23-35), 4 están separadas y 1 casada. Todas tienen hijos entre 1 y 3 . Tres mujeres tienen a los hijos con ellas y 2 en sus países de origen al cuidado de los abuelos maternos. Tienen estudios secundarios, trabajan en el servicio doméstico y cuidado de ancianos y ganan menos de $1.000 €$ mensuales con ingresos variables. Llevan casi 3 años fuera de sus países $(M$ $=35,8$ meses, $D T=22,86$ meses) y tienen una deuda media de $2.100 €(D T=3.911,52 €)$. Comparten piso con otras personas, 1 persona comparte habitación con otra persona, 2 viven con su pareja e hijos en una única habitación y 2 tienen habitación propia. Ninguna de las mujeres tiene antecedentes psiquiátricos, pero 3 han sido víctimas de maltrato en su infancia y adolescencia y 2 han sido víctimas de agresión aquí, ninguna toma psicofármacos. Desde el punto de vista de su situación legal, ninguna posee permiso de residencia y trabajo permanente, 2 tienen permisos provisionales y 3 no tienen ni permiso de residencia ni de trabajo, y una de ellas no está empadronada ni posee tarjeta sanitaria cuando acude al programa.

\section{Diseño}

El estudio se ha realizado con un diseño de caso único A-B más seguimiento de 1 y 3 meses.

\section{Hipótesis}

- El programa de apoyo psicológico resultará eficaz para la disminución de los síntomas, la consecución de los objetivos y la mejora de la autoestima.

- La mejoría lograda en el postratamiento se mantendrá en los seguimientos.

\section{Instrumentos de evaluación}

Para las variables sociodemográficas y migratorias, se ha llevado a cabo una entrevista inicial, donde se obtenían datos referidos, por un lado, a variables demográficas y, por otro, a variables migratorias analizadas en este trabajo: edad, sexo, estado civil, vivienda, procedencia y fecha de salida, situación familiar, formación académica, situación laboral y económica, situación legal, así como al estado de salud (historia psiquiátrica anterior, enfermedades y posesión de tarjeta sanitaria).

Symptom Checklist-90-R (Derogatis, 1975; versión española de González de Rivera, 2002). Se utilizó para evaluar la presencia de síntomas psicopatológicos generales. La escala consta de 90 ítems, con 5 alternativas de respuesta en una escala de tipo Likert, que 
oscilan entre 0 (nada) y 4 (mucho). La escala evalúa 9 dimensiones de síntomas (somatización, obsesión-compulsión, sensibilidad interpersonal, depresión, ansiedad, hostilidad, ansiedad fóbica, ideación paranoide y psicoticismo). Ofrece además 3 índices globales que reflejan el malestar del sujeto y que son el índice global de severidad (GSI), el índice total de síntomas positivos (PST) y el índice de la intensidad de los síntomas (PSDI). La consistencia interna del cuestionario oscila entre 0,81 y 0,90 y la estabilidad temporal entre 0,78 y 0,90 .

Escala de Autoestima (Rosenberg, 1965). Tiene como objetivo evaluar el sentimiento de satisfacción que una persona tiene consigo misma. Este instrumento consta de 10 ítems generales que puntúan de 1 a 4 en una escala de tipo Likert. El rango del cuestionario es de 10 a 40, con una puntuación tanto mayor cuanto mayor es la autoestima. La fiabilidad test-retest es de 0,85 y el coeficiente alfa de consistencia interna de 0,92 .

Escala de Conductas-Objetivo (Echeburúa y Corral, 1987). Es un autoinforme diseñado para que los pacientes concreten una lista de cinco conductas que quieren mejorar y supondrían un beneficio importante para su vida cotidiana. Estas cinco conductas se valoran según su grado de dificultad de 1 a 10.

Cuestionario de Satisfacción con el Trata- miento (Larsen, Attkinson, Hargreaves y Nguyen, 1979). Consta de 8 ítems relacionados con la calidad de la atención terapéutica, con la intensidad de la ayuda recibida y con la satisfacción con el tratamiento. Los ítems están estructurados en una escala de tipo Likert, 5 formulados en forma positiva y 3 en forma negativa para el control de la aquiescencia, cada uno con una puntuación de 1 a 4 . El rango del cuestionario es de 8 a 32 .

\section{Programa de tratamiento}

El programa de apoyo psicológico, consta de 8 sesiones semanales, de hora y media de duración cada sesión, con un formato individual y de tipo cognitivo-conductual. Tiene un enfoque centrado en la solución de problemas e intenta abarcar la sintomatología ansioso-depresivasomática y confusional y enseñar a las inmigrantes habilidades para hacer frente al estrés, y a las dificultades que tienen en su vida cotidiana. El objetivo general sería fortalecer a las inmigrantes y dotarles de estrategias para paliar el malestar psicológico que padecen y ayudarlas a conseguir sus objetivos vitales. El programa incluye tareas para casa y la entrega de un cuaderno de trabajo. En la Tabla 1 se expone un resumen de los componentes específicos del programa.

Tabla 1. Componentes del programa de apoyo psicológico

- Apoyo emocional y facilitación del desahogo, relato de su vida y su historia migratoria.

- Psicoeducación sobre las implicaciones psicológicas de la emigración y los síntomas más importantes.

- Síntomas ansiosos: técnicas de respiración y relajación.

- Hábitos de sueño y alimentación.

- Mejora del estado de ánimo: realización de actividades positivas y manejo de pensamientos negativos.

- Mejora de la autoestima e identificación de fortalezas.

- Mejora de las relaciones sociales.

- Prevención de recaídas.

\section{Procedimiento}

Se divulgó el programa de apoyo psicológico a inmigrantes en las instituciones que trabajan directamente con esta población (Cruz Roja, Caritas, SOS-Racismo, Asesoría jurídica y legal del Gobierno Vasco para inmigrantes HELDU y trabajadores sociales) y en los locutorios. En la divulgación se entregaron marca-páginas con la dirección y el teléfono al que podían llamar los inmigrantes para solicitar la ayuda. Cuando un inmigrante llamaba, se le daba cita para rea- 
lizar la primera entrevista y en ella se explicaba el programa y los objetivos, se entregaba una hoja informativa descriptiva del programa y una carta de consentimiento y se pasaba a realizar la entrevista inicial y en el caso de que la paciente estuviera de acuerdo se procedía a la evaluación pretratamiento que podía oscilar entre 2 y 3 sesiones. Posteriormente se aplicó el programa de apoyo psicológico y al finalizarlo se realizó la evaluación postratamiento y las posteriores evaluaciones de seguimiento al mes y a los 3 meses. Toda la intervención, tanto la evaluación como el tratamiento, fue llevada a cabo por una psicóloga clínica con más de 10 años de experiencia.

\section{Análisis}

Se realizaron análisis estadísticos con el SPSS-16. Se analizaron las medias agrupadas y se calculó el tamaño del efecto en el postratamiento y en el seguimiento de los 3 meses usando delta (la diferencia de la media del pre-tratamiento menos la media del post-tratamiento o de los seguimientos dividiendo por la desviación típica de las puntuaciones del pretratamiento). Además se compararon los resultados con los baremos españoles del SCL90-R de la población general y psiquiátrica.

A nivel individual se realizó un análisis del cambio clínicamente significativo utilizando la fórmula de Jacobson y Truax (1991) del índice de cambio fiable (ICF) para determinar si la magnitud del cambio para un sujeto es estadísticamente significativa en el postratamiento y en el seguimiento de los 3 meses. El índice de cambio fiable es calculado para cada sujeto restando las puntuaciones del pretest y del postest o de los seguimientos y dividiendo por el error típico de la diferencia entre las puntuaciones de las dos aplicaciones de la prueba (Ddif).

$$
\begin{aligned}
& \text { ICF }=\text { pre-post } / \text { Ddif } \\
& \text { Ddif }=\sqrt{ } 2(\mathrm{DE})^{2} \\
& \mathrm{DE}=D T \text { pre } \sqrt{(1-r \text { pre-post })}
\end{aligned}
$$

Un ICF mayor de 1.96 es poco probable ( $p$ $<0,05)$ que ocurra sin un cambio realmente significativo.

\section{RESULTADOS}

A continuación se presentan los resultados previos al programa en las variables psicopatológicas, de autoestima y conductas objetivo, y posteriormente los resultados de las participantes tras el programa de apoyo psicológico en la evaluación postratamiento y en los seguimientos.

\section{Resultados en variables psicopatológicas, de autoestima y conductas-objetivo}

A continuación se presentan los resultados en la escala SCL-90-R, en la escala de autoestima y en la escala de conductas-objetivo obtenidas en la evaluación realizada antes de aplicar el programa de apoyo psicológico.

El nivel de malestar psicológico de las inmigrantes es muy alto en todas las subescalas, pero es realmente significativo, en las escalas de depresión $(2,67)$, sensibilidad interpersonal $(2,16)$, somatización $(2,04)$, obsesión-compulsión $(1,92)$, ansiedad $(1,80)$ e ideación paranoide $(1,65)$. Estas puntuaciones indican un nivel de gravedad del malestar psicológico intenso. Presentan intensos sentimientos de soledad, tristeza, de sentirse atrapadas, desesperanzadas y con la sensación de que todo requiere un gran esfuerzo. Las puntuaciones en la subescala de sensibilidad interpersonal señalan las dificultades en las relaciones, la incomodidad, la timidez, la sensación de sentirse incomprendida que pueden derivarse del choque cultural y del poco tiempo de residencia en Guipúzcoa. Las puntuaciones elevadas en la subescala de somatización señalan la presencia de dolores de cabeza, dolores musculares, de debilidad y sufrimiento psicosomático. Aparecen reflejadas en la subescala de obsesión-compulsión, por un lado, las preocupaciones, las dificultades de concentración, de memoria, de toma de decisiones a nivel cognitivo y por otro, a nivel conductual, la necesidad de repetir y de comprobar que realizan las cosas de modo correcto sin cometer errores, ya que su situación de vulnerabilidad no se lo permite. Respecto a la ideación paranoide muestran gran desconfianza y temor hacia los demás derivado de la situación de indefensión e ilegalidad en la que viven. 
Tabla 2. Resultados en el pretratamiento y cambios en los síntomas psicopatológicos, en la autoestima y en las conductas-objetivo

\begin{tabular}{|c|c|c|c|c|c|c|c|c|}
\hline \multirow{2}{*}{ Variables } & \multicolumn{2}{|c|}{ Pretratamiento } & \multicolumn{2}{|c|}{ Postratamiento } & \multicolumn{2}{|c|}{$1 \mathrm{mes}$} & \multicolumn{2}{|c|}{3 meses } \\
\hline & $M$ & (DT) & $M$ & $(D T)$ & $M$ & $(D T)$ & $M$ & (DT) \\
\hline \multicolumn{9}{|l|}{ SCL-90-R } \\
\hline Somatización & 2,04 & $(0,53)$ & 0,43 & $(0,33)$ & 0,58 & $(0,35)$ & 0,41 & $(0,24)$ \\
\hline Obsesión-Compulsión & 1,92 & $(0,64)$ & 0,80 & $(0,73)$ & 0,77 & $(0,84)$ & 0,55 & $(0,38)$ \\
\hline Sensibilidad Interpersonal & 2,16 & $(0,92)$ & 0,66 & $(0,57)$ & 0,59 & $(0,85)$ & 0,52 & $(0,36)$ \\
\hline Depresión & 2,67 & $(0,55)$ & 0,96 & $(0,71)$ & 0,82 & $(0,73)$ & 0,55 & $(0,23)$ \\
\hline Ansiedad & 1,80 & $(0,77)$ & 0,48 & $(0,54)$ & 0,62 & $(0,62)$ & 0,50 & $(0,34)$ \\
\hline Hostilidad & 1,25 & $(1,31)$ & 0,25 & $(0,34)$ & 0,15 & $(0,18)$ & 0,56 & $(0,75)$ \\
\hline Ansiedad fóbica & 1,02 & $(0,92)$ & 0,51 & $(0,70)$ & 0,17 & $(0,26)$ & 0,34 & $(0,27)$ \\
\hline Ideación Paranoide & 1,65 & $(0,71)$ & 0,78 & $(0,72)$ & 0,66 & $(0,79)$ & 0,62 & $(0,31)$ \\
\hline Psicoticismo & 1,12 & $(0,71)$ & 0,38 & $(0,49)$ & 0,30 & $(0,47)$ & 0,30 & $(0,18)$ \\
\hline GSI & 1,88 & $(0,53)$ & 0,59 & $(0,54)$ & 0,57 & $(0,59)$ & 0,48 & $(0,29)$ \\
\hline PST & 65,40 & $(15,51)$ & 32 & $(20,26)$ & 28,75 & $(20,40)$ & 34,5 & $(20,98)$ \\
\hline PSDI & 2,57 & $(0,34)$ & 1,43 & $(0,53)$ & 1,50 & $(0,67)$ & 1,09 & $(0,59)$ \\
\hline Autoestima (rango: 10-40) & 29 & $(7,1)$ & 34,4 & $(4,5)$ & 35,25 & $(4,57)$ & 34 & $(2,16)$ \\
\hline Conductas objetivo (rango: 5-50) & 46,6 & $(0,89)$ & 15,4 & $(7,53)$ & 15,5 & $(5,91)$ & 9,5 & $(3,31)$ \\
\hline
\end{tabular}

Nota. GSI: índice global de severidad; PST: índice total de síntomas positivos; PSDI: índice de la intensidad de los síntomas.

En las medidas generales de la escala de SCL-90-R, las puntuaciones indican gran intensidad de sufrimiento $(\mathrm{GSI}=1,88)$, una gran amplitud y diversidad de la psicopatología (PST $=65,4)$ y una alta intensidad de los síntomas (PSDI $=2,57$ ). Estas mujeres presentan un sufrimiento psicológico muy intenso.

En la escala de autoestima las puntuaciones son moderadas altas, es decir las mujeres no tienen una mala imagen de sí mismas, a pesar de todas las dificultades que tienen. En la escala de conductas objetivo perciben sus objetivos con un nivel de dificultad muy alto.

En la Tabla 3 se exponen brevemente algunas de las conductas-objetivo que las mujeres han señalado para trabajar a lo largo de las sesiones del programa de apoyo psicológico. En general, las conductas-objetivo tienen relación principalmente con la ansiedad, la depresión y la confusión. Y, además, se incluyen aspectos

Tabla 3. Ejemplos de conductas objetivo a trabajar en el programa

- Ser más fuerte, tener menos miedo y enfrentarse a las situaciones.

- Tener más seguridad y confianza en una misma, más autoestima.

- Tener menos preocupaciones, poder centrarse en las cosas.

- Manejar la ansiedad y el nerviosismo.

- Manejar la timidez y la vergüenza.

- Mejorar la tristeza, tener más ánimo y sentirse menos culpable.

- Reducir el perfeccionismo.

- Mejorar las relaciones personales con la pareja, con la familia, en la sociedad.

- Saber decir que no, defender derechos, pedir algo.

- No gritar, manejar la ira, la rabia y la frustración.

- Poder estudiar.

- Poder tener papeles, es decir, los permisos de trabajo y residencia. 
como la mejora en las relaciones sociales, la reducción de la ira y el perfeccionismo y el logro de objetivos vitales, tales como poder estudiar y regularizar su situación.

Cambios en las variables psicopatológicas, de autoestima y en las conductas-objetivo

A nivel general, teniendo en cuenta todos los indicadores, las mujeres experimentan tras el programa de apoyo psicológico una clara mejoría en la sintomatología psicopatológica. Esta mejoría es muy evidente entre la evaluación pretratamiento y postratamiento, y los logros obtenidos se mantienen en la evaluación del mes y de los 3 meses de seguimiento (Tabla 2).

Los cambios en la escala de autoestima no son tan apreciables ya que el nivel en el pretratamiento ya era moderado-alto, a pesar de ello, el nivel de autoestima también mejora.

Respecto a las conductas objetivo que las pacientes se marcaron antes de iniciar el programa, el nivel de dificultad que ellas perciben para lograr sus objetivos vitales y mejorar su vida se reduce considerablemente entre el pretratamiento y el postratamiento, y entre éste y el seguimiento de los 3 meses sigue reduciéndose. Lo cual indica que la mejoría sigue aumentando.

Además en la escala de satisfacción con el tratamiento con un rango de 8-32, obtienen una media de 31,6 $(D T=0,54)$ que indica un nivel de satisfacción muy alto con la ayuda recibida.

Desde el punto de vista del tamaño del efecto en la Tabla 4 se muestran los resultados del efecto de la intervención en el postratamiento y a los tres meses de seguimiento.

En el postratamiento los tamaños del efecto son mayores de 0,7 establecido por Cohen, excepto en ansiedad fóbica. En el resto de las medidas se aprecian cambios importantes entre el pretratamiento y el postratamiento. A los 3 meses todos los tamaños del efecto son mayores de 0,7 , lo que indica también una clara mejoría, excepto en hostilidad. En líneas generales los tamaños del efecto conseguidos en el postratamiento se mantienen o incluso aumentan en el seguimiento de los tres meses en todas las medidas lo que indica una evidente mejoría terapéutica.
Tabla 4. Tamaño del efecto

\begin{tabular}{lcc}
\hline Variables & Pre-post & Pre-3 meses \\
\hline SCL-90-R & 3,03 & 3,07 \\
Somatización & 1,75 & 2,14 \\
Obsesión-Compulsión & 1,63 & 1,78 \\
Sensibilidad interpersonal & 3,10 & 3,85 \\
Depresión & 1,71 & 1,68 \\
Ansiedad & 0,76 & 0,52 \\
Hostilidad & 0,55 & 0,73 \\
Ansiedad fóbica & 1,22 & 1,45 \\
Ideación paranoide & 1,04 & 1,15 \\
Psicoticismo & 2,43 & 2,64 \\
\hline GSI & 2,15 & 1,99 \\
PST & 3,35 & 4,35 \\
PSDI & 0,76 & 0,70 \\
\hline Autoestima (rango: 10-40) & 35,05 & 41,68 \\
Conductas objetivo (rango: 5-50)
\end{tabular}

Nota. GSI: índice global de severidad; PST: índice total de síntomas positivos; PSDI: índice de la intensidad de los síntomas.

\section{Comparaciones con baremos españoles de población normal y psiquiátrica en el SCL-90-R}

A continuación se muestran las diferencias entre las puntuaciones a lo largo de las evaluaciones teniendo en cuenta los baremos españoles de la escala SCL-90-R.

Teniendo en cuenta el baremo de la población general no clínica, las pacientes de la muestra en la evaluación pretratamiento superan ampliamente el percentil 90 en todas las medidas y subescalas; sin embargo en la evaluación realizada tras participar en el programa de apoyo psicológico a los 3 meses de finalización del mismo, se puede ver que las puntuaciones se reducen considerablemente con respecto a la población no clínica, situándose alrededor del percentil 50, si se exceptúan las escalas de ideación paranoide, psicoticismo, ansiedad fóbica y hostilidad que están un poco por encima de la media.

Estos resultados pueden ser comprendidos teniendo en cuenta la situación de miedo, vulnerabilidad y peligrosidad real que viven las 
Tabla 5. Comparaciones entre las puntuaciones de la muestra y los baremos españoles del SCL-90-R

\begin{tabular}{lcccccc}
\hline \multicolumn{1}{c}{ Variables } & $\begin{array}{c}\text { Pre } \\
M\end{array}$ & $\begin{array}{c}\text { Percentil } \\
\text { población } \\
\text { general }\end{array}$ & $\begin{array}{c}\text { Percentil } \\
\text { población } \\
\text { psiquiátrica }\end{array}$ & $\begin{array}{c}3 \text { meses } \\
M\end{array}$ & $\begin{array}{c}\text { Percentil } \\
\text { población } \\
\text { general }\end{array}$ & $\begin{array}{c}\text { Percentil } \\
\text { población } \\
\text { psiquiátrica }\end{array}$ \\
\hline GSI & 1,88 & 99 & 65 & 0,48 & 50 & 5 \\
PST & 65,4 & 99 & 60 & 34,5 & 70 & 15 \\
PSDI & 2,57 & 95 & 55 & 1,09 & 5 & 5 \\
\hline Subescalas somatización & 2,04 & 97 & 55 & 0,41 & 40 & 10 \\
Obsesión compulsión & 1,92 & 95 & 55 & 0,55 & 55 & 10 \\
Sensibilidad interpersonal & 2,16 & 99 & 75 & 0,52 & 60 & 15 \\
Depresión & 2,67 & 99 & 75 & 0,55 & 35 & 5 \\
Ansiedad & 1,80 & 97 & 60 & 0,50 & 55 & 10 \\
Hostilidad & 1,25 & 90 & 60 & 0,56 & 65 & 30 \\
Ansiedad fóbica & 1,02 & 90 & 55 & 0,34 & 65 & 25 \\
Ideación Paranoide & 1,65 & 97 & 65 & 0,62 & 70 & 25 \\
Psicoticismo & 1,12 & 95 & 60 & 0,30 & 70 & 20 \\
\hline
\end{tabular}

Nota. GSI: índice global de severidad; PST: índice total de síntomas positivos; PSDI: índice de la intensidad de los síntomas.

pacientes. Así, no es extraño que piensen que no se pueden fiar de la gente, muchas han tenido experiencias desagradables, o que tengan la sensación de que se les mira, ya que realmente es así (su aspecto físico es diferente y su entonación también), o que crean que los demás se van a aprovechar de ellas. Estos pensamientos que componen la subescala de ideación paranoide evidencian la situación de desconfianza en la que viven. Las puntuaciones en la subescala de psicoticismo remiten a la profunda sensación de soledad, de sentirse distante y diferente de la gente y a pensar que hay algo que no funciona adecuadamente en su mente, las pacientes señalan que no se habían sentido tan confusas nunca. Las puntuaciones en la subescala de ansiedad fóbica hablan de miedo y en la subescala de hostilidad, de agresividad, irritabilidad e ira contenida, fruto de las situaciones injustas en las que viven.

Teniendo en cuenta el baremo de la población psiquiátrica (grupo formado por pacientes psiquiátricos ambulatorios con diagnósticos ansiosos y depresivos fundamentalmente) las puntuaciones de estas mujeres en la evaluación pretratamiento se sitúan alrededor del percentil 60 , lo que indica un nivel de sintomatología psicopatológica bastante alto, con puntuaciones más altas que el percentil 75 en depresión y sensibilidad interpersonal. Tras recibir el programa de apoyo psicológico y en la evaluación de los 3 meses, las puntuaciones se reducen considerablemente siendo el percentil 30 en la subescala de hostilidad, el más alto. Estos resultados indican la eficacia del programa para ayudar a estas mujeres, a sentirse mejor y a reducir sus síntomas.

\section{Análisis de los cambios individuales clínicamente significativos}

Desde el punto de vista individual del análisis del cambio clínicamente significativo en la Tabla 6 se muestran los ICF en el posttratamiento y en el seguimiento de los 3 meses de la medida global del SCL-90-R (GSI).

Desde el punto de vista de la intensidad del sufrimiento por los síntomas psicopatológicos la mejoría obtenida por las pacientes en el postratamiento es importante en todos los casos excepto en la paciente 3 . A los tres meses todas las pacientes consiguen cambios positivos importantes e incluso algunas de ellas paciente1, paciente 2 y paciente 4 se podrían considerar recuperadas. 
Tabla 6. Índice de cambio fiable

\begin{tabular}{cccccccccccc}
\hline & \multicolumn{2}{c}{ Paciente 1} & \multicolumn{2}{c}{ Paciente 2} & \multicolumn{2}{c}{ Paciente 3} & \multicolumn{2}{c}{ Paciente 4} & \multicolumn{2}{c}{ Paciente 5 } \\
\cline { 2 - 13 } Variable & Pre & Pre & Pre & Pre & Pre & Pre & Pre & Pre & Pre & Pre \\
& Post & 3 meses & Post & 3 meses & Post & 3 meses & Post & 3 meses & Post & 3 meses \\
\hline GSI & 1,61 & 2,63 & 3,01 & 2,98 & 0,93 & 1,31 & 3,28 & 2,74 & 1,36 & 1,56 \\
(SCL-90-R) & & $\mathrm{R}$ & $\mathrm{R}$ & $\mathrm{R}$ & & & $\mathrm{R}$ & $\mathrm{R}$ & & \\
\hline
\end{tabular}

Nota. GSI: índice global de severidad. Cambio menor de 1: mejoría sin recuperación total. Cambio mayor de 1 y menor de 1,96: cambio positivo importante. Cambio mayor de 1,96: recuperado (R).

\section{DISCUSIÓN}

Los diferentes estudios señalan que emigrar supone un proceso altamente estresante para una persona. Sin embargo, apenas existen programas de asistencia primaria que aborden esta situación psicológica, de manera que permitan mejorarla y prevenir la posible aparición de trastornos mentales y es difícil también que los inmigrantes soliciten ayuda en los programas de apoyo psicológico por la desconfianza y el desconocimiento (Fernando, 1995). Por estas razones se planteó el diseño y la evaluación de un programa de apoyo psicológico a inmigrantes.

A pesar de las limitaciones de este estudio, ya que la muestra sólo es de 5 casos y los baremos de la escala de psicopatología general son españoles y no latinoamericanos, los resultados muestran que se da una clara mejoría entre la evaluación pretratamiento y la realizada después de recibir el programa de apoyo psicológico. Además esta mejoría se mantiene y aumenta entre esta evaluación y la realizada a los 3 meses. Así la mejoría clínica obtenida por estas mujeres es evidente y puede ser atribuida al programa ya que no hay una mejora en su situación legal, ni laboral, ni económica en el plazo en el que se lleva a cabo el estudio. Resultados similares se encontraron en un estudio realizado con mujeres mexicanas víctimas de violencia doméstica con un programa breve de 8 sesiones (Labrador y Alonso, 2007).

La situación psicológica en la que acuden estas mujeres al programa es de un nivel de sufrimiento muy alto. Teniendo en cuenta las puntuaciones en la escala de psicopatología general SCL-90-R, las mujeres presentan en el pretratamiento niveles muy altos de depresión, ansiedad, somatización y confusión, lo que confirmaría la presencia de un síndrome relacionado con las situaciones de estrés crónico y múltiple de los inmigrantes. Además comparando sus puntuaciones previas al programa con los baremos se aprecia un nivel de malestar alto tal y como señalaban en su estudio Valiente, Sandín, Chorot, Santed y González de Rivera (1996). Otra característica importante de estas mujeres es que más de la mitad han sufrido maltrato en su país, dato que es corroborado en otros estudios (Labrador y Alonso, 2007) y que se añade al sufrimiento de emigrar. Asimismo, las mujeres acuden a solicitar ayuda en la primera fase de asentamiento, cuando llevan menos de 5 años fuera de sus países, y cuando quizá más síntomas presentan (Hovey y Magaña, 2000).

El programa de apoyo psicológico funciona como un dispositivo de salud mental primario, un sistema de apoyo, contención y de aprendizaje de estrategias psicológicas para hacer frente a las dificultades cotidianas de las mujeres. Además ayuda a éstas a conseguir aquellos objetivos psicológicos de bienestar que se proponen al acudir al programa y por lo tanto a integrarse mejor en la sociedad receptora.

La reducción en la sintomatología es muy evidente en la mayoría de las escalas del SCL90-R, quizá lo es menos en las escalas de psicoticismo, ideación paranoide y hostilidad, ya que estas dimensiones no fueron específicamente tratadas en el programa de apoyo psicológico. Sin embargo, y a pesar de esto, las pacientes también mejoraron en estos índices, así como en la autoestima y en las conductas objetivo. Lo que demuestra la utilidad de un programa breve, estructurado y sencillo para mejorar la salud psicológica de las mujeres inmigrantes y que podría ser llevado a 
cabo en los niveles de asistencia primaria. Además, las pacientes muestran un nivel de satisfacción muy alto con el apoyo recibido.

A pesar de la situación económica, laboral y legal en la que se encuentran estas personas y que no puede ser modificada por una intervención psicológica, lo cierto es que ésta les ayuda y les da poder para continuar luchando por su supervivencia y la de sus familias. El programa sirve de apoyo en la primera fase de asentamiento y podría servir como dispositivo de salud que impide la cronificación o el empeoramiento de la sintomatología que presentan las mujeres inmigrantes.

Un reto de futuro, teniendo en cuenta las limitaciones de este trabajo, sería ampliar el estudio con un mayor número de participantes y un seguimiento más largo (12 meses), para comprobar si el programa resulta de utilidad también a largo plazo. Así como, aplicar el programa en diferentes tipos de inmigrantes, incluir un grupo de control o comparar el programa de apoyo psicológico aplicado individualmente o de modo grupal. Tal y como señala Watters (2002) es necesario conocer la salud mental de los inmigrantes, promover servicios que les ayuden a mejorar su salud y mejorar los existentes y su acceso, ya que los datos señalan que no cubren sus necesidades.

\section{REFERENCIAS}

Achotegui, J. (2000). Los duelos de la emigración. Jano, psiquiatría y humanidades, 2, 15-19.

Achotegui, J. (2002). La depresión en los inmigrantes: una perspectiva transcultural. Barcelona: Ediciones Mayo.

Achotegui, J. (2003). Depresión y ansiedad en los inmigrantes. Barcelona: Ediciones Mayo.

Alma, W. (1986). Psychosocial problems of migrants. En M. Colledge, y P.G. Svensson (Eds.), Migration and health. La Haya: OMS.

Basabe, N., Zlobina, A., y Páez, D. (2004). Integración socio-cultural y adaptación psicológica de los inmigrantes extranjeros en el País Vasco. Cuadernos Sociológicos Vascos, 15.

Bernal, M. (2002). Good practice in mental health and social care provisions for immigrants, asylum seekers and refugees in Spain. Servicio de Atención Psicopatológica y Psicosocial a Inmigrantes y Refugiados SAPPIR. (http://www.migra-salut-mental-org).
Berry, J.W. (2001). A psychology of immigration. Journal of Social Issues, 57, 615-631.

Berry, J.W. (2005). Acculturation: living successfully in two cultures. International Journal of Intercultural Relations, 29, 697-712.

Colegio Oficial de Psicólogos (1994). Guía de apoyo para el profesional de la intervención social con inmigrantes económicos y refugiados, 1 . Madrid.

Derogatis, L.R. (1975). The SCL-90-R. Baltimore: Clinical Psychometric Research.

Echeburúa, E., y Corral, P. (1987). Escala de conductas objetivo. Manuscrito no publicado.

Fernando, S. (1995). Mental health in a multi-ethnic society: a multi-disciplinary handbook. London: Routledge.

Flaherty, J.A., Kohn, R., Levav, I., y Birz, B. (1988). Demoralization in soviet-jewish immigrants to the United States and Israel. Comprehensive Psychiatry, 29, 558-597.

García-Campayo, J. (2000). Psiquiatría transcultural: el desafío de la psicosomática en el nuevo milenio. Cuadernos de Medicina Psicosomática, 54, 5-6.

García-Campayo, J., y Sanz, C. (2002). Salud mental en inmigrantes el nuevo desafío. Medicina Clínica, 118, 187-191.

Garza-Guerrero, A.C. (1974). Culture shock: it's mourning and the vicisitudes of identity. Journal of American Psychoanalitical Association, 22, 408-429.

González de Rivera, J.L. (2002). SCL-90-R. Cuestionario de 90 síntomas. Adaptación española. Madrid: TEA

González, M.T., y Landero, R. (2007). Cuestionario de afrontamiento del estrés (CAE): validación en una muestra mexicana. Revista de Psicopatología y Psicología Clínica, 12, 189-198.

Hovey, J.D. (2001). Mental health and substance abuse. Program for the study of immigration and mental health. Universidad de Toledo.

Hovey, J.D., y Magaña, C. (2000). Acculturative stress, anxiety and depression among immigrant farmworkers in the midwest. Journal of Immigrant Health, 2, 119131.

Instituto Nacional de Estadística (2008). Avance del Padrón Municipal a 1 de enero de 2008. Datos provisionales. (http://www.ine.es)

Jansá, J.M., y García de Olalla, P. (2004). Salud e inmigración: nuevas realidades y nuevos retos. Gaceta Sanitaria, 18, 207-213.

Jarvis, G., Kirmayer, L., Weinfeld, M., y Lasry, J. (2005). Religious practice and psychological distress: the importance of gender, ethnicity and immigrant status. Transcultural Psychiatry, 42, 657-675.

Kareem, J., y Littlewood, R. (2000). Intercultural therapy. London: Blackwell Science.

Labrador, F.J., y Alonso, E. (2007). Eficacia a corto plazo de un programa de intervención para el trastorno de estrés postraumático en mujeres mexicanas víctimas 
de violencia doméstica. Revista de Psicopatología y Psicología clínica, 12, 117-130.

Larsen, D., Attkinson, C., Hargreaves, W., y Nguyen, T. (1979). Assessment of client/patient satisfaction: development of a general scale. Evaluation and Program Planning, 2, 197-207.

Lerner, Y., Kertes, J., y Zilber, N. (2005). Immigrants from the former Soviet Union, 5 years post-immigration to Israel: adaptation and risk factors for psychological distress. Psychological Medicine, 35, 1805-1814.

Mallona, A. (1999). Surfacing the self: narratives of central American immigrant women. Dissertation Abstract International Behavior Science England, 59, 4510.

Marsen, E. (2004). Arteterapia e inmigración. Revista de Treball Social, 173, 71-76.

Marsella, A.J., y Pedersen, P. (2004). Internationalizing the counselling psychology curriculum: toward new values, competencias and directions. Counselling PSyhology Quarterly, 17, 413-423.

Martínez, M.F., García, M., y Maya, I. (1999). El papel de los recursos sociales naturales en el proceso migratorio. Intervención Psicosocial, 8, 221-232.

Martínez-Taboada, C., Arnoso, A., y Elgorriaga, E. (2006). Estudio del choque psicosocial de las personas inmigradas en Donostia. Síntesis y Propuestas. San Sebastián: Ayuntamiento de San Sebastián.

Matamala, P., y Crespo, A. (2004). Pacientes inmigrantes en un Centro de Salud Mental Comunitario. VII congreso virtual de psiquiatría. (http://www.interpsiquis. $\mathrm{com} / 2004)$

Observatorio Vasco de Inmigración (2007). Tablas sobre población en territorios históricos de la CAPV y su porcentaje de extranjeros. (http://www.ikuspegi.org)

Pernice, R., y Brook, J. (1996). The mental health pattern of migrants: is there a euphoric period followed by a mental health crisis? International Journal Social Psychiatry, 42, 18-27.

Petry, N., Armentano, C., Kuoch, T., Norinth, T., y Smith, L. (2003). Gambling participation and problems among South East Asian refugees to the United States. Psychiatric Services, 54, 1142-1148.

Pumariega, A.J., Rothe, E., y Pumariega, J.B. (2005). Mental health of immigrants and refugees. Community Mental Health Journal, 41, 581-597.
Ramos, P.J., y León, A. (2007). La medida del síndrome de Ulises. Ansiedad y estrés, 13, 253-268.

Ritsner, M., y Ponizovsky, A. (1999). Psychological distress through immigration: the two-phase temporal pattern? International Journal of Social Psychiatry, 45, 125-139.

Ritsner, M., Ponizovsky, A., y Ginath, Y. (1997). Changing patterns of distress during the adjustment of recent immigrants: a 1-year follow-up study. Acta Psychiatrica Scandinavica, 95, 494-499.

Rosenberg, A. (1965). Society and the adolescent selfimage. New Jersey: Princenton.

Salvador, I. (2001). La emigración como proceso de duelo. Protocolo de psicoterapia breve centrada en su elaboración. Revista Española de Neuropsiquiatría, 82, 57-64.

Scott, W.A., y Scott, R. (1985). Some predictors of migrant adaptation available at selection time. Australian Psychologist, 20, 313-343.

Steele, L., Lemieux-Charles, L., Clark, J., y Glazier, R.H. (2002). The impact of policy changes on the health of recent immigrants and refugees in the inner city. A qualitative study of service provedeers' perspectives. Canadian Journal of Public Health, 93, 118-122.

Talarn, A., Navarro, N., Rosell, L., y Rigat, A. (2006). Propuesta de especificadores diagnósticos vinculados al estrés y el trauma: una aportación a la nosología psicopatológica. Revista de Psicopatología y Psicología clínica, 11, 107-114.

Tizón, J.L. (1994). Emigrar: soñar, sufrir... tal vez elaborar, tal vez crear. Revista de Treball Social, 136, 22-38.

Valiente, R.M., Sandín, B., Chorot, P., Santed, M.A., y González de Rivera, J.L. (1996). Sucesos vitales mayores y estrés: efectos psicopatológicos asociados al cambio por migración. Psiquis, 17, 211-230.

Villar, V. (2002). Migración extracomunitaria: la realidad que no existe. Educación Social, 20, 99-113.

Walsh, S., Shulman, S., Feldman, B., y Maurer, O. (2005). The impact of immigration on the internal processes and developmental tasks of emerging adulthood. Journal of Youth and Adolescence, 34, 413-426.

Watters, CH. (2002). Migration and mental health care in Europe: report of a preliminary zapping exercise. Journal of Ethnic and Migration Studies, 28, 153-172. 